\title{
Microstructural modeling of collagen network mechanics and interactions with the proteoglycan gel in articular cartilage
}

Received: 15 July 2005 / Accepted: 21 September 2005 / Published online: 20 May 2006

(c) Springer-Verlag 2006

\begin{abstract}
Cartilage matrix mechanical function is largely determined by interactions between the collagen fibrillar network and the proteoglycan gel. Although the molecular physics of these matrix constituents have been characterized and modern imaging methods are capable of localized measurement of molecular densities and orientation distributions, theoretical tools for using this information for prediction of cartilage mechanical behavior are lacking. We introduce a means to model collagen network contributions to cartilage mechanics based upon accessible microstructural information (fibril density and orientation distributions) and which self-consistently follows changes in microstructural geometry with matrix deformations. The interplay between the molecular physics of the collagen network and the proteoglycan gel is scaled up to determine matrix material properties, with features such as collagen fibril pre-stress in free-swelling cartilage emerging naturally and without introduction of ad hoc parameters. Methods are developed for theoretical treatment of the collagen network as a continuum-like distribution of fibrils, such that mechanical analysis of the network may be simplified by consideration of the spherical harmonic components of functions of the fibril orientation, strain, and stress distributions. Expressions for the collagen network contributions to matrix stress and stiffness tensors are derived, illustrating that only spherical harmonic components of orders 0 and 2 contribute to the stress, while orders 0,2 , and 4 contribute to the stiffness. Depth- and compression-dependent equilibrium mechanical properties of cartilage matrix are modeled, and advantages of the approach are illustrated by exploration of orientation and strain distributions of collagen fibrils in compressed cartilage. Results highlight collagen-proteoglycan interactions, especially for very small physiological strains where experimental data are
\end{abstract}

T. M. Quinn ( $\varangle) \cdot$ V. Morel

Cartilage Biomechanics Group,

Ecole Polytechnique Fédérale de Lausanne (EPFL), EPFL Station 15,

AI 1234, CH-1015 Lausanne, Switzerland

E-mail: Thomas.Quinn@epfl.ch

Tel.: +41-21-6938350

Fax: +41-21-6938660 relatively sparse. These methods for determining matrix mechanical properties from measurable quantities at the microscale (composition, structure, and molecular physics) may be useful for investigating cartilage structure-function relationships relevant to load-bearing, injury, and repair.

\section{Introduction}

Articular cartilage is a composite polyelectrolyte hydrogel with mechanical properties determined by interactions among its molecular constituents (Grodzinsky, 1983). The major constituents are a collagen fibrillar network and a proteoglycan (PG) gel. The molecular physics of these individual components have been characterized by a range of investigations. Tensile properties of collagen molecules (Luo et al. 2004) have been observed experimentally, in addition to the properties of collagen fibers (Pins et al. 1997) which form the mechanically functional network. In theoretical modeling, collagen mechanics is often addressed using rope-like constitutive laws with spring-like behavior in tension and negligible resistance in compression (Farquhar et al. 1990; Soulhat et al. 1999; Wilson et al. 2004). The PG gel provides much resistance to fluid flow (Zamparo and Comper 1989) and solute transport (Torzilli et al. 1997) in cartilage. Furthermore, it accounts for most of the matrix fixed charge density (Maroudas et al. 1969; Bashir et al. 1999), which contributes to electrokinetic phenomena (Frank and Grodzinsky 1987) and compressive stiffness (Seog et al. 2002). Theoretical models of PG gel molecular physics including electrokinetic transport (Eisenberg and Grodzinsky 1988), electrostatic component of compressive modulus (Buschmann and Grodzinsky 1995), and anisotropic hydraulic permeability (Quinn et al. 2001a) have been developed through extensions of unit cell approaches for transport in fibrous media (Happel 1959; Kuwabara 1959; Brenner and Edwards 1993). Molecular scale models of collagen and PG contributions to cartilage mechanics are useful for relating the compression-dependent 
material properties of articular cartilage (Mow et al. 1984; Chen et al. 2001; Quinn et al. 2001b; Reynaud and Quinn 2006) to microstructural changes which occur during tissue deformations (Quinn et al. 2001a).

Interactions between collagen network tensile properties and PG gel swelling pressure contribute to cartilage mechanics and matrix microstructural organization (Maroudas 1976; Williams et al. 1996). The mechanics of cartilage explant disks in radially unconfined axial compression (Armstrong et al. 1984; Kim et al. 1995) involve predominant contributions from PG gel swelling pressures for axial stresses, balanced by collagen network tensile stresses in the radial direction. These contributions combine to determine the tissue compressive Young's modulus $(E)$ and Poisson's ratio ( $v)$ at equilibrium (Jurvelin et al. 1997). PG gel flow resistance introduces transient fluid pressurization, which together with collagen viscoelasticity (Wilson et al. 2004) can influence time-dependent behavior (Mizrahi et al. 1986; Jurvelin et al. 1997) important to cartilage biomechanical function (Park et al. 2003) and injury (Morel and Quinn 2004a).

Collagen contributions to cartilage mechanics depend upon network organization, molecular physics, and tissue deformation. Collagen type II molecules are organized into a crosslinked fibrillar network in cartilage. Fibrillar size depends upon matrix location at cell and tissue length scales. The pericellular matrix contains a fine collagen latticework while the interterritorial matrix between cells contains a mixed population of relatively large fibrils together with a finer latticework (Hunziker et al. 1997). Furthermore, different tissue zones exhibit different collagen densities (Camacho et al. 2001) and predominant orientations of large interterritorial fibrils. Consistent with classical arcade-like descriptions of collagen network organization in cartilage (Benninghoff 1925), the superficial zone contains fibrils oriented primarily parallel to the articular surface (Jeffery et al. 1991), while deep tissue zones may exhibit large interterritorial fibrils tending to be oriented perpendicular to the bone-cartilage interface (Hunziker et al. 1997), with the intermediate zone containing more random fibril orientations (Mollenhauer et al. 2003). The depth-dependent structure of the collagen network likely underlies depth-dependence of cartilage mechanical behavior (Chen et al. 2001). Estimates of the mechanical constitutive behavior of collagen fibrils have suggested ropelike behavior with tensile moduli up to $750 \mathrm{MPa}$, depending upon the degree of crosslinking (Pins et al. 1997). Collagen network structural organization changes as the tissue deforms (Kääb et al. 1998; Alhadlaq and Xia 2004). Therefore, measurement of collagen fibril densities and orientations (Camacho et al. 2001; Xia et al. 2001; Xia and Elder 2001; Mollenhauer et al. 2003) might be applicable to estimation of local matrix mechanical properties, if links between molecular physics, matrix structural organization, and tissue deformations are established.

Several theoretical models have been developed to address collagen network mechanics. Scale-up from molecular physics to tissue properties, with individual fibrils subject to continuum-scale deformations and network mechanics determined from superposition of many individual fibrils has been explored in the contexts of cartilage (Farquhar et al. 1990), tissue-equivalent materials (Barocas and Tranquillo 1997), and aortic valves (Billiar and Sacks 2000; Driessen et al. 2003). The "fibril-reinforced" model for articular cartilage aimed to explicitly address the mechanics of the collagen network and the PG gel acting simultaneously (Soulhat et al. 1999). In this particular approach, collagen fibril microstructural details were ignored and replaced by nonlinear spring elements representing network constitutive behavior. As a result, this approach relied upon empirical characterization in specific loading configurations (rather than independently acquired molecular physics and microstructural data) for modeling of collagen network mechanics. Furthermore, the PG gel was modeled as poroelastic (Soulhat et al. 1999; Li et al. 2000) instead of a gas-like, flow-resistant material consistent with the understanding of its molecular physics (Grodzinsky 1983; Buschmann and Grodzinsky 1995). Therefore, the "fibril-reinforced" approach does not fully separate collagen and PG contributions to cartilage mechanics: the PG gel possesses a seemingly nonphysical Poisson's ratio and collagen fibrils support zero tensile stress under tissue free-swelling conditions, which is inaccurate (Maroudas 1976). Limitations of the "fibril-reinforced" model have been partially addressed by extensions to include greater numbers of spring elements representing collagen network constitutive behavior (Wilson et al. 2004) and more careful attention to interactions between collagen tension and PG gel swelling pressures in the absence of tissue loading (Wilson et al. 2005). However, these approaches still rely upon empirical characterization of the effective constitutive behavior of matrix constituents in specific loading configurations, and retain ad hoc parameters such as PG gel Poisson's ratio. Therefore they are limited in their potential for estimation of cartilage mechanical properties from independently determined microstructural data and molecular physics.

Our objectives were to develop a constitutive model of the cartilage collagen network based upon measurable structural quantities including fibril density and orientation distributions. This microstructure-based approach aims for direct scale-up from structural organization and physics at the molecular scale to mechanics at the tissue scale, without case-specific empirical characterization. It therefore may be readily applied to matrix from any tissue zone or extracellular location, and any mechanical loading conditions. For implementation in finite element calculations of cartilage deformations proceeding with incremental time steps, constitutive laws governing matrix poroelastic mechanics are derived from microstructural data. Small deformations are allowed to occur, then associated microstructural changes are determined and constitutive laws re-derived. Therefore modeled deformations proceed as many small linear steps, but microstructural data and matrix properties are continuously updated to model nonlinear behavior over large deformations. 


\section{Methods}

Cartilage matrix elements large compared to the molecular scale but small enough compared to the tissue scale to have uniform composition, structure, and physical properties were considered. Each element was assumed to have two components: a collagen network and a PG gel (Fig. 1). The collagen network consisted of collagen fibrils and their hydration water (Maroudas and Bannon 1981), while the PG gel consisted of proteoglycans, their hydration water, and water which could flow during matrix deformations. With the volume fraction of PG gel represented by $\gamma$, that for the collagen network was $1-\gamma$.

Matrix elastic moduli were determined from changes to the elastic stress tensor in response to small deformations. Small matrix deformations relative to a reference state were described by a vector displacement field $\mathbf{u}$; its gradient decomposes into the symmetric strain tensor $(\mathbf{E})$ and an antisymmetric component $(\boldsymbol{\Omega})$ representing solid body rotation (Malvern 1969); respectively

$\nabla \mathbf{u}=\frac{1}{2}\left(\nabla \mathbf{u}+(\nabla \mathbf{u})^{t}\right)+\frac{1}{2}\left(\nabla \mathbf{u}-(\nabla \mathbf{u})^{t}\right)=\mathbf{E}+\mathbf{\Omega}$

where superscript $t$ denotes transposition. The equilibrium elastic stress tensor for the cartilage matrix element (S) changed with deformation. This transition was expressed in terms of $\mathbf{E}$ and $\boldsymbol{\Omega}$ to define the fourth-order matrix stiffness tensor J (Farquhar et al. 1990):

$\mathbf{S}_{d}=\mathbf{S}+\mathbf{J}: \mathbf{E}+\mathbf{R}: \mathbf{\Omega}$

where subscript $d$ denotes a quantity after a small deformation and $\mathbf{R}: \boldsymbol{\Omega}$ accounts for pure rotation without stiffness.

The total equilibrium elastic stress tensor (S) for the cartilage matrix element was the sum of the area-averaged equilibrium elastic stress tensors of the PG gel $\left(\mathbf{S}_{\mathrm{pg}}\right)$ and collagen network $\left(\mathbf{S}_{\mathrm{col}}\right)$. Using Eq. 2, separate stiffness tensors for the PG gel $\left(\mathbf{J}_{\mathrm{pg}}\right)$ and collagen network $\left(\mathbf{J}_{\mathrm{col}}\right)$ were defined such that $\mathbf{J}=\mathbf{J}_{\mathrm{pg}}+\mathbf{J}_{\mathrm{col}}$. However, this was complicated by

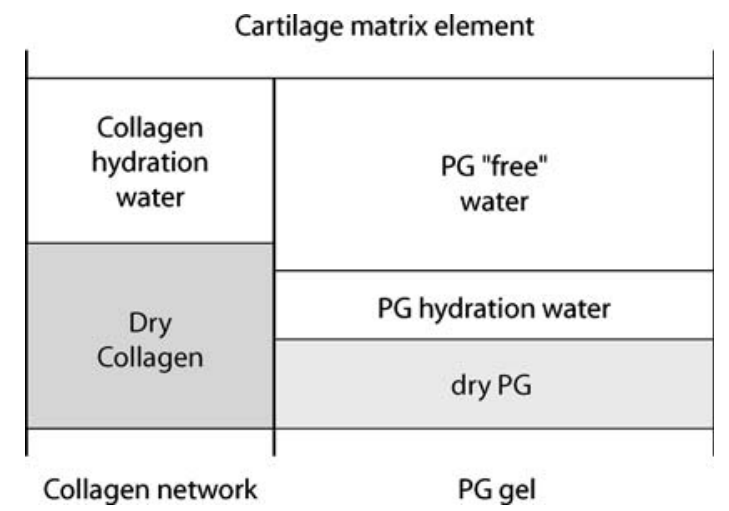

Fig. 1 Cartilage matrix elements are considered to contain a collagen network component and a PG gel component. The collagen network consists of dry collagen and bound hydration water, and is considered to conserve its volume during tissue deformations. The PG gel consists of proteoglycans, their hydration water, and freely movable water differential effects of deformation on each matrix component. Collagen fibrils were assumed subject to the same displacement field $\mathbf{u}$ as the matrix itself at the continuum scale (below), but the collagen network was assumed to conserve its volume (fibrils and collagen hydration water; Fig. 1). Therefore, matrix element volume changes required relatively greater volume changes in the $\mathrm{PG}$ gel. The strain tensor E can be decomposed into isotropic and deviatoric (D) components (Malvern 1969), respectively

$$
\begin{aligned}
\mathbf{E} & =\mathbf{I} \frac{\nabla \cdot \mathbf{u}}{3}+\left[\frac{1}{2}\left(\nabla \mathbf{u}+(\nabla \mathbf{u})^{t}\right)-\mathbf{I} \frac{\nabla \cdot \mathbf{u}}{3}\right] \\
& =\mathbf{I} \frac{\nabla \cdot \mathbf{u}}{3}+\mathbf{D}
\end{aligned}
$$

where I represents the unit dyadic tensor. For small deformations, the isotropic part results in a fractional change of total element volume of $\nabla \cdot \mathbf{u}$ while $\mathbf{D}$ involves no volume change. The fractional change of PG gel volume was therefore $\gamma^{-1} \nabla \cdot \mathbf{u}$. With $\alpha$ representing the PG gel "solid" (dry PG plus hydration water) volume fraction, the changes of $\gamma$ and $\alpha$ during small deformations were

$\gamma_{d}=\gamma+(1-\gamma) \nabla \cdot \mathbf{u}$

and

$\alpha_{d}=\alpha\left(1-\gamma^{-1} \nabla \cdot \mathbf{u}\right)$

The equilibrium elastic stress tensor for the PG gel was determined from Poisson-Boltzmann modeling of the electrostatic contribution to swelling pressure of glycosaminoglycan solutions (Buschmann and Grodzinsky 1995) using a 20-node finite difference calculation implemented on a spreadsheet. Consistent with previous results, $0.25 \mathrm{MPa}$ was added to the electrostatic contribution to estimate total PG gel swelling pressure (Buschmann and Grodzinsky 1995). Results were indistinguishable from previous findings and summarized by

$\mathbf{S}_{\mathrm{pg}}[\mathrm{MPa}]=\gamma\left[274.1 \alpha^{2}-15.98 \alpha+0.27733\right] \mathbf{I}$

$\mathbf{S}_{\mathrm{pg}}$ was therefore always isotropic in the present model so that anisotropic mechanics could only arise due to contributions from the collagen network. Eq. 4, 5, and 6 provide

$$
\begin{aligned}
\mathbf{J}_{p g}[\mathrm{MPa}]=- & {\left[274.1 \alpha^{2}(1+\gamma)\right.} \\
& -15.98 \alpha \gamma-0.02733(1-\gamma)] \mathbf{I I}
\end{aligned}
$$

for the PG gel contribution to the matrix stiffness tensor.

Collagen fibrils were assumed the basic unit of construction of the collagen network, with larger fibers equivalent to many fibrils oriented in the same direction. It was assumed that fibrils were of uniform size (constant volume), and that a single constitutive law relating fibril tensile stress $\sigma$ to strain $\varepsilon_{f}$ could be identified. It was also assumed that for any direction the mean fibril number density could be defined (with opposite directions sharing equally). To determine the areaaveraged stress tensor of the collagen network $\left(\mathbf{S}_{\mathrm{col}}\right)$, consider a matrix volume of length $d_{\text {tot }}$ and cross-sectional area $A_{\text {tot }}$ across which an imaginary test surface with unit normal $\mathbf{n}$ is drawn (Fig. 2a). The probability of interaction with the test 

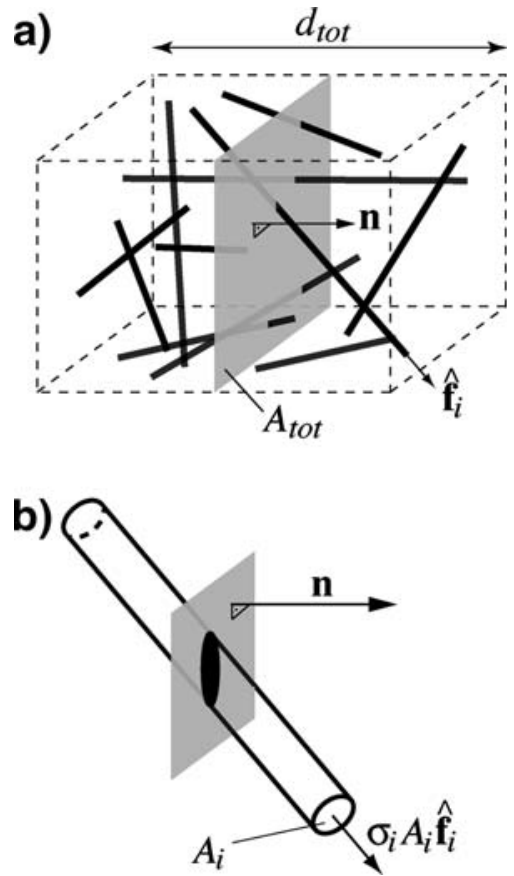

Fig. 2 a Sketch of matrix control volume containing collagen fibers (black bars). For each fiber orientation $\left(\hat{\mathbf{f}}_{i}\right)$, a single fiber stress $\left(\sigma_{i}\right)$ is assumed. The stress tensor for the collagen network is determined by considering forces acting through a control surface drawn (gray) through the matrix. b Close-up of an imaginary control surface drawn through an individual collagen fiber of cross-sectional area $A_{i}$, for which the contribution to the collagen network stress tensor is to be determined. The tensile force in the fiber is given by $\sigma_{i} A_{i} \hat{\mathbf{f}}_{i}$

surface for a fibril with a given orientation (labeled with subscript $i$ ) is $\mathbf{f}_{i} \cdot \mathbf{n} / d_{\text {tot }}$, where $\mathbf{f}$ is a vector representing fibril length and direction. Since the tensile force associated with each fibril is $\sigma_{i} A_{i} \hat{\mathbf{f}}_{i}$ where $\hat{\mathbf{f}}_{i}=\mathbf{f}_{i} /\left|\mathbf{f}_{i}\right|$ and $A_{i}$ represents fibril cross-sectional area (Fig. 2b), the average stress due to collagen fibrils acting across the test surface is

$$
\begin{aligned}
\mathbf{S}_{\mathrm{col}} \cdot \mathbf{n} & =\frac{1}{A_{\mathrm{tot}}} \sum_{i} N_{i}\left(\frac{\mathbf{f}_{i} \cdot \mathbf{n}}{d_{\mathrm{tot}}}\right) \sigma_{i} A_{i} \hat{\mathbf{f}}_{i} \\
& =\sum_{i} \frac{N_{i}}{N_{\mathrm{tot}}} \sigma_{i}\left(\frac{A_{i}\left|\mathbf{f}_{i}\right| N_{\mathrm{tot}}}{A_{\mathrm{tot}} d_{\mathrm{tot}}}\right) \hat{\mathbf{f}}_{i} \hat{\mathbf{f}}_{i} \cdot \mathbf{n}
\end{aligned}
$$

where $N_{i}$ is the number of fibrils with a given orientation and $N_{\text {tot }}$ is the total number of fibrils in the test volume. Since fibrils were assumed to conserve volume, the term in brackets on the right side of Eq. 8 is the fibril volume fraction $(1-\gamma)$. With $P(\theta, \phi) \sin \theta \mathrm{d} \theta \mathrm{d} \phi$ representing the fraction of fibers oriented between $\theta$ and $\theta+\mathrm{d} \theta$, and $\phi$ and $\phi+\mathrm{d} \phi, \mathbf{S}_{\mathrm{col}}$ may therefore be expressed as the integral

$\mathbf{S}_{\mathrm{col}}=(1-\gamma) \int_{0}^{2 \pi} \int_{0}^{\pi} P(\theta, \phi) \sigma(\theta, \phi) \mathbf{i}_{R} \mathbf{i}_{R} \sin \theta \mathrm{d} \theta \mathrm{d} \phi$

where $R, \theta, \phi$ have their usual meanings in spherical coordinates.
Under a small displacement field $\mathbf{u}$, vectors representing fibrils (f) were assumed to undergo a continuum-scale mapping.

$\mathbf{f}_{d}=\mathbf{f} \cdot(\mathbf{I}+\nabla \mathbf{u})$.

When following an individual fibril during deformation in the Lagrangian sense, tensile strains and stresses varied as

$\varepsilon_{\mathrm{fd}}=\varepsilon_{f}+\left(1+\varepsilon_{f}\right) \mathbf{i}_{R} \mathbf{i}_{R}: \nabla \mathbf{u}$

$\sigma_{d}=\sigma+\frac{\partial \sigma}{\partial \varepsilon_{f}}\left(1+\varepsilon_{f}\right) \mathbf{i}_{R} \mathbf{i}_{R}: \nabla \mathbf{u}$

where $\mathbf{i}_{R}$ denotes a unit radial vector defining fibril orientation. Equations 4, 9, 10, and 12 were used to determine $\mathbf{J}_{\text {col }}$ (Morel 2004), which may be written

$\mathbf{J}_{\mathrm{col}}=-\mathbf{S}_{\mathrm{col}} \mathbf{I}+\mathbf{T}_{\mathrm{col}}+\mathbf{R}_{\mathrm{col}}$

where

$\mathbf{T}_{\mathrm{col}}=(1-\gamma) \int_{0}^{2 \pi} \int_{0}^{\pi} P(\theta, \phi)\left[\frac{\partial \sigma}{\partial \varepsilon_{f}}\left(1+\varepsilon_{f}\right)-2 \sigma\right]$

$$
\times \mathbf{i}_{R} \mathbf{i}_{R} \mathbf{i}_{R} \mathbf{i}_{R} \sin \theta \mathrm{d} \theta \mathrm{d} \phi
$$

and $\mathbf{R}_{\mathrm{col}}={ }^{\text {col }} \mathbf{R}_{i j k l}$ is most easily expressed with index notation as

${ }^{\mathrm{col}} \mathrm{R}_{i j k l}={ }^{\mathrm{col}} \mathrm{S}_{i l} \delta_{j k}+{ }^{\mathrm{col}} \mathrm{S}_{j l} \delta_{i k}$

where ${ }^{\mathrm{col}} \mathrm{S}_{i j}=\mathbf{S}_{\mathrm{col}}$ and $\delta_{i j}$ is the Kronecker delta function. Equations 9 and 14 therefore prescribe calculations for determination of collagen network contributions to the matrix stiffness tensor (Eq. 13), provided $\gamma, P(\theta, \phi), \varepsilon_{f}(\theta, \phi)$, and $\sigma\left(\varepsilon_{f}\right)$ are known.

With the poroelastic properties of cartilage matrix elements determined from collagen network and PG gel microstructure, small deformations under loading may be modeled and microstructural changes in matrix architecture calculated. To first order in $\mathbf{u}$, Eqs. 4 and 5 describe changes in $\gamma$ and $\alpha$, which completely characterize PG gel microstructure for present purposes. Equation 11 describes changes in individual fibril strains in the Lagrangian sense, but requires modification to

$$
\begin{aligned}
\varepsilon_{\mathrm{fd}}(\theta, \phi)= & \varepsilon_{f}(\theta, \phi)+\left(1+\varepsilon_{f}(\theta, \phi)\right) \mathbf{i}_{R} \mathbf{i}_{R}: \nabla \mathbf{u} \\
& -\mathbf{i}_{R} \cdot \nabla \mathbf{u} \cdot \nabla \varepsilon_{f}(\theta, \phi)
\end{aligned}
$$

for description of changes in $\varepsilon_{f}(\theta, \phi)$ in the laboratory frame. The fibril orientation distribution $P(\theta, \phi)$ varied as

$$
\begin{aligned}
P_{d}(\theta, \phi)= & P(\theta, \phi)\left[1-\left(\mathbf{I}-3 \mathbf{i}_{R} \mathbf{i}_{R}\right): \nabla \mathbf{u}\right] \\
& -\mathbf{i}_{R} \cdot \nabla \mathbf{u} \cdot \nabla P(\theta, \phi)
\end{aligned}
$$

accurate to first-order terms in $\mathbf{u}$ (Morel 2004).

In practice, the forms of Eq. 9 and 14 make it efficient to work in terms of the spherical harmonic decompositions (SHDs) of $P(\theta, \phi), \varepsilon_{f}(\theta, \phi)$, and $\sigma(\theta, \phi)$ which characterize collagen network microstructure. The tensor $\mathbf{i}_{R} \mathbf{i}_{R}$ only contains spherical harmonic components of orders 0 and 2; therefore due to orthogonality of spherical harmonic functions only the SHD of the product $P(\theta, \phi) \sigma(\theta, \phi)$ of orders 
0 and 2 contribute to $\mathbf{S}$ (Eq. 9). This permits integration to be performed "in advance", and Eq. 9 may be replaced by a dot product between vectors representing the amplitudes of the SHD of $P(\theta, \phi) \sigma(\theta, \phi)$ and the results of Eq. 9 for individual spherical harmonic functions in place of $P(\theta, \phi) \sigma(\theta, \phi)$. Similarly, $\mathbf{i}_{R} \mathbf{i}_{R} \mathbf{i}_{R} \mathbf{i}_{R}$ only contains spherical harmonic components of orders 0, 2, and 4, and Eq. 14 may be replaced by a dot product between vectors representing the amplitudes of the SHD of $P(\theta, \phi)\left[\frac{\partial \sigma}{\partial \varepsilon_{f}}\left(1+\varepsilon_{f}\right)-2 \sigma\right]$ and the results of Eq. 14 for individual spherical harmonic functions in its place. Similarly, the forms of Eq. 16 and 17 and the properties of spherical harmonics make it convenient to calculate deformation-induced changes in $P(\theta, \phi)$ and $\varepsilon_{f}(\theta, \phi)$ while remaining in the SHD domain (Morel 2004).

A piecewise linear rope-like constitutive law was assumed for all fibrils,

$$
\begin{array}{ll}
\sigma=M \varepsilon_{f} & \text { if } \varepsilon_{f} \geq 0 \\
\sigma=0 & \text { if } \varepsilon_{f}<0
\end{array}
$$

with a constant tensile modulus of elasticity $M=70 \mathrm{MPa}$ corresponding to lightly crosslinked fibrils from previous measurements (Pins et al. 1997). For small changes from freeswelling matrix geometry, where all collagen fibers remain in tension, mathematical linearity permits the full calculation "cycle" (Fig. 3) to remain entirely in the SHD domain (Morel 2004). For modeling of matrix strains in excess of a few percent, it becomes convenient to return to the spatial domain for determination of $\sigma(\theta, \phi)$ from $\varepsilon_{f}(\theta, \phi)$. For this purpose, the code ccSHT was implemented, kindly provided by Christopher Cantalupo.

Superficial, intermediate, and deep tissue zones were modeled (Fig. 4) using previously determined values (Maroudas et al. 1991; Buschmann and Grodzinsky 1995) for freeswelling matrix composition and microstructure (Table 1). Spherical harmonic components up to order 6 were included in calculations. To compare mechanical behaviors among the three zones, simulations were performed corresponding to radially unconfined static axial compression of cartilage disks between rigid, frictionless, impermeable boundaries. The "axial" direction of compression was perpendicular to the articular surface. For each simulation, the cartilage disk had uniform composition corresponding to one of the defined tissue zones (Table 1), thereby representing a homogeneous tissue slice. Under these conditions, the equilibrium mechanical properties of an individual element corresponded to those of an entire tissue zone. This relatively simplified approach was taken in order to examine the abilities of the modeling approach to realistically predict matrix mechanics from microstructural data, and to differentiate between the mechanics of tissue zones with different microstructural organization.

\section{Results}

Under free-swelling conditions, the absence of externally applied loads implied that isotropic PG gel swelling pressure was balanced solely by tensile forces in the collagen network.

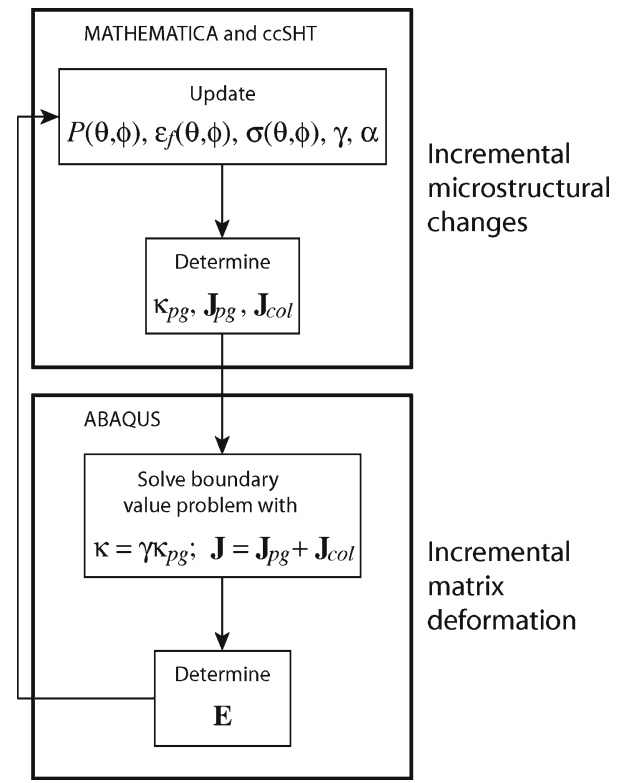

Fig. 3 Outline of the calculation scheme for model implementation. Initial conditions for matrix microstructural parameters $\left(P(\theta, \phi), \varepsilon_{f}(\theta, \phi), \gamma, \alpha\right)$ were estimated from literature values and used to determine matrix mechanical properties $\left(\boldsymbol{\kappa}_{\mathrm{pg}}, \mathbf{J}_{\mathrm{pg}}, \mathbf{J}_{\mathrm{col}}\right)$. Properties were then input to a finite element model where small matrix deformations were allowed to occur subject to applied loads. These small deformations $(\mathbf{E})$ were then used to determine changes in matrix microstructural parameters and to update the matrix mechanical properties prior to finite element calculation of the next small deformation

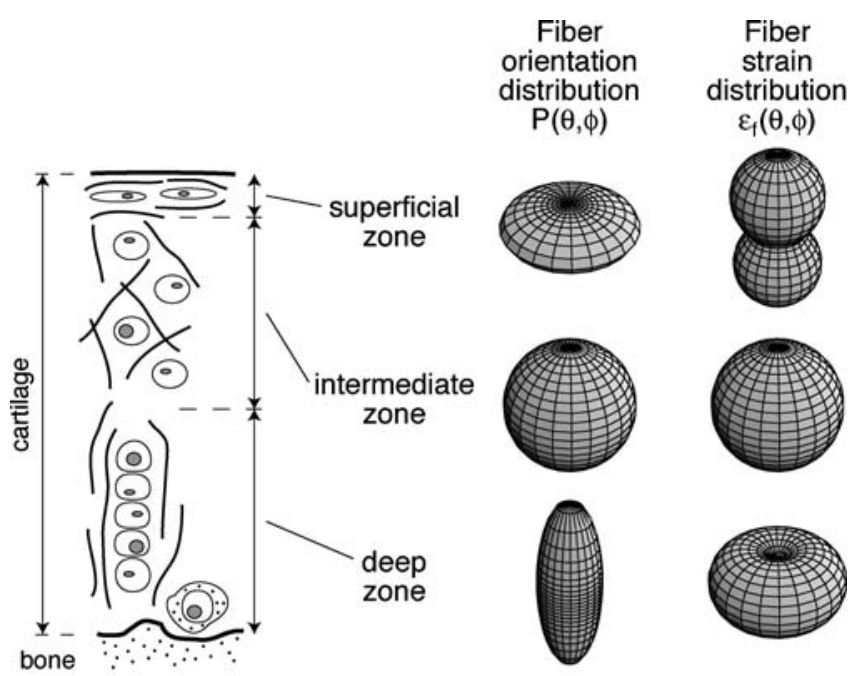

Fig. 4 Orientation distributions of collagen fibers in superficial, intermediate, and deep zones of cartilage under free-swelling conditions. Corresponding strains within individual collagen fibrils are also expressed as a function of fibril orientation

In this situation, different cartilage zones exhibited markedly different distributions of collagen fibril loading (Fig. 4). In the superficial zone, where the network consisted of fibrils with orientations preferentially parallel to the articular surface, PG gel swelling pressure was balanced by a greater number of fibrils in these directions as compared to other 
Table 1 Modeling parameters characterizing microstructure of cartilage zones for free-swelling conditions prior to applied deformations

\begin{tabular}{|c|c|c|c|c|c|c|}
\hline \multirow[t]{2}{*}{ Tissue zone } & \multirow[t]{2}{*}{$\begin{array}{l}\text { Matrix volume } \\
\text { fraction of PG gel }(\gamma)\end{array}$} & \multirow[t]{2}{*}{$\begin{array}{l}\text { PG gel volume } \\
\text { fraction of solid }(\alpha)\end{array}$} & \multicolumn{4}{|c|}{$\begin{array}{l}\text { Collagen fibril orientation distribution: } \\
\text { coefficients of spherical harmonic components }\end{array}$} \\
\hline & & & $\mathrm{Y}_{0}^{0}$ & $\mathrm{Y}_{2}^{0}$ & $\mathrm{Y}_{4}^{0}$ & $\mathrm{Y}_{6}^{0}$ \\
\hline Superficial & 0.55 & 0.06 & 0.2821 & -0.0899 & 0.0351 & 0.0149 \\
\hline Intermediate & 0.70 & 0.12 & 0.2821 & 0.0000 & 0.0000 & 0.0000 \\
\hline Deep & 0.65 & 0.13 & 0.2821 & 0.0810 & 0.0307 & 0.0129 \\
\hline
\end{tabular}

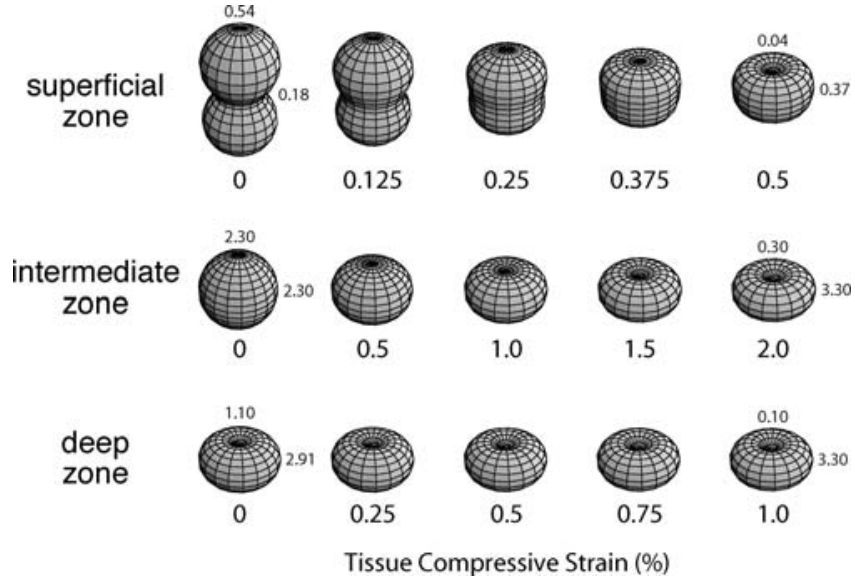

Fig. 5 Strains within individual collagen fibrils $\varepsilon_{f}(\theta, \phi)$ during small quasistatic compressions of superficial, intermediate, and deep tissue zones. For each zone, calculations were performed for compression ranges where fibril strains remained positive for all directions. Quantitative values for strains (expressed as percent) in fibrils oriented parallel and perpendicular to the compression direction are written above and beside strain distributions

directions. As a consequence, individual fibrils oriented perpendicular to the articular surface supported greater tensile loads than those oriented in parallel directions, since fewer fibrils were available (per unit surface) to balance the same swelling pressure. In the intermediate zone, where collagen fibril orientations were distributed uniformly in all directions, tensile stresses in individual fibrils were likewise identical in all directions. In the deep zone, the network consisted of fibrils oriented preferentially in the direction perpendicular to the interface with underlying bone. Stresses within individual fibrils in the deep zone were therefore greatest for fibrils oriented parallel to the bone interface, under free-swelling conditions.

In the presence of externally applied loads, forces within the two matrix components no longer balanced each other. Instead, matrix deformations occurred such that at mechanical equilibrium the net contribution of the PG gel and the collagen network balanced the external loads. In the superficial zone, tensile stresses in fibrils oriented perpendicular to the articular surface were relieved by unconfined compression (Fig. 5), while a combination of changes occurred in the collagen network allowing increased tensile load support in directions parallel to the articular surface. First, individual fibrils oriented parallel to the articular surface supported greater loads. Second, fibrils tended to change their orientations toward these directions, increasing the capacity of the collagen network to support tensile loads perpendicular to the compression direction. Third, as the fluid was expelled from the PG gel during compression, the volume fraction of collagen fibrils $(1-\gamma)$ increased, providing increased tensile load support per unit area of matrix (Eq. 9). These collagen network structural changes occurred to offset increases in the swelling pressure of the PG gel resulting from its volume reduction during matrix compression. Similar changes in both matrix components occurred within the intermediate and deep zones, although in each case these changes emerged from the zone-specific distributions of collagen fibril stresses existing prior to compression.

For comparison with previous experimental results, equilibrium matrix mechanical properties were also calculated for each tissue zone near to free-swelling conditions. At $0.5 \%$ compression, Young's moduli were $2.8 \mathrm{MPa}$ in the superficial zone, $3.5 \mathrm{MPa}$ in the intermediate zone, and $5.9 \mathrm{MPa}$ in the deep zone, while Poisson's ratios were 0.36, 0.40, and 0.42 , respectively (Table 2 ). With increasing compression, Young's moduli tend to increase while Poisson's ratios decreased (Table 2).

\section{Discussion}

This work aims to bridge gaps between histological data for cartilage structure and theoretical tools for simulating cartilage function. The approach builds upon previous work by introducing means for treating the collagen network as a continuum-like distribution of fibrils rather than a collection of a small number of representative spring elements. Advantages of this new approach include the derivation of general relationships for collagen network contributions to cartilage mechanics (Eqs. 9, 13, and 14), and retaining a basis for collagen network modeling (fibril densities and orientation distributions) which follows straightforwardly from independent structural measurements. Matrix material properties are determined simply from the mechanical interplay between the collagen network and the PG gel (Maroudas 1976), providing a means for scale-up from molecular physics to matrix properties without introduction of ad hoc parameters such as Poisson's ratio of the PG gel (Soulhat et al. 1999; Wilson et al. 2004). As a result, clear distinctions between PG gel and collagen network contributions to matrix mechanics are obtained, with features such as collagen fibril pre-stress in free-swelling cartilage emerging naturally. This model therefore translates directly between measurable quantities at the microscale (composition, structure, and molecular physics) 
Table 2 Model results for cartilage matrix mechanical properties in different cartilage zones, for compression regimes where all collagen fibrils remained in tension

\begin{tabular}{|l|l|l|l|l|l|l|}
\hline Tissue Zone & \multicolumn{2}{|c|}{$\begin{array}{c}\text { Young's modulus } \\
(\mathrm{MPa})\end{array}$} & \multicolumn{3}{c|}{ Poisson's ratio } \\
& \multicolumn{3}{|c|}{$\begin{array}{l}\text { Matrix } \\
\text { compression }\end{array}$} & \multicolumn{3}{c|}{$\begin{array}{l}\text { Matrix } \\
\text { compression }\end{array}$} \\
\hline & $0.5 \%$ & $1.0 \%$ & $2.0 \%$ & $0.5 \%$ & $1.0 \%$ & $2.0 \%$ \\
\hline Superficial & 2.8 & & & 0.36 & & \\
\hline Intermediate & 3.5 & 3.6 & 3.7 & 0.40 & 0.37 & 0.32 \\
\hline Deep & 5.9 & 6.0 & & 0.42 & 0.39 & \\
\hline
\end{tabular}

and at the macroscale (matrix material properties), which may be useful for investigating cartilage structure-function relationships in many contexts.

Limitations of this approach include potential inaccuracies due to overt reliance upon microstructure-based models of matrix molecular physics. The constitutive behavior of collagen fibrils depends upon fibrillar structure including crosslinking (Pins et al. 1997), so that definition of a fibrillar "structural unit" from which all larger fibrils are composed is questionable. As suggested by previous work (Wilson et al. 2005), it may be more accurate to consider the collagen network as a superposition of multiple networks with different fibril sizes and constitutive behaviors. Unit cell models of PG gel molecular physics are based upon several simplifying assumptions and therefore imperfect by their nature (Buschmann and Grodzinsky 1995). In order to emphasize the emergence of collagen network mechanics from individual fibrillar behavior, the present model deliberately neglected some aspects of molecular physics which may be important under certain conditions, such as collagen fibril viscoelasticity (Wilson et al. 2004) and PG gel anisotropic permeability (Reynaud and Quinn 2006). Furthermore, the cartilage matrix contains many constituents in addition to fibrillar collagens and PGs; these minor constituents may contribute to matrix mechanics directly or by modifying the behavior and interactions of the two major constituents.

Findings for equilibrium mechanical properties near freeswelling conditions within different cartilage matrix zones were reasonably consistent with previous experimental findings. Poisson's ratios were in the range of $0.32-0.42$ (Table 1), somewhat elevated compared to previous measurements (Jurvelin et al. 1997). This discrepancy appeared to be due to the very small compressive strains modeled, where radial deformations of axially compressed explant disks could occur more easily due to collagen reorientation as opposed to the much stiffer fibril extension predominant at the higher strains of experiments. Modeled Poisson's ratios increased with depth in cartilage consistent with depth-dependent deformations of full-thickness explant disks (Jurvelin et al. 1997). Modeled Young's moduli also increased with depth within cartilage (Table 2) consistent with previous findings (Chen et al. 2001). Values of Young's moduli ranged from 2.8 to 6.0 MPa which were elevated by approximately $10 \times$ compared to typical experimental results (Jurvelin et al. 1997; Chen et al. 2001). However, this discrepancy has a rational basis which highlights mechanical interactions between the collagen network and the PG gel. Very small compressive strains in the range of 1-2\% involve many collagen fibrils going from extended to relaxed, such that PG gel swelling pressures must be compensated by external forces over a small range of strain. As a result, matrix compressive stiffness can be greatly elevated compared to that of the PG gel alone (Fig. 6). Experimental measurements of cartilage stiffness are typically performed for matrix compressive strains $\geq 5 \%$, where changes in PG gel swelling pressure dominate mechanical behavior and matrix stiffness is approximately $0.5 \mathrm{MPa}$ (as suggested by Eq. 6 and Table 1). Therefore, mechanical characterization techniques which can assay matrix behavior in the range of $1 \%$ compressive strains are of significant interest for exploring collagen-PG interactions. The present model provides reasonable results for cartilage equilibrium mechanical properties, for compositional and structural parameters corresponding to matrix superficial, intermediate, and deep zones. In addition, modeled changes in matrix microstructure were consistent with previous observations (Kääb et al. 1998), providing a clear illustration of links between molecular organization and tissue-scale mechanical properties in cartilage.

Previous experimental studies of cartilage mechanical behavior may be better interpreted in light of the present model. Radially confined axial compression of cartilage disks resulted in increases of matrix swelling pressure which were more dramatic in the axial versus the radial direction (Khalsa and Eisenberg 1997). This was considered surprising because these changes were presumed to result from swelling pressure increases within the PG gel alone, and were expected to be isotropic. However, collagen network volume fraction $(1-\gamma)$ increases during radially confined axial compression, providing increased tensile load support per unit area (Eq. 9) in the radial direction while it decreases in the axial direction (Fig. 5), consistent with the observed behavior (Khalsa and Eisenberg 1997). Nevertheless, radial confinement of axially compressed cartilage disks may prevent collagen network contributions to offsetting PG gel swelling pressure by other mechanisms which are operative without confinement (such as increased deformation in the radial direction). Other experimental studies have highlighted unconfined radial deformations of axially compressed cartilage disks which were greater for directions perpendicular versus parallel to the superficial zone split-line pattern (Mizrahi et al. 1986). The 

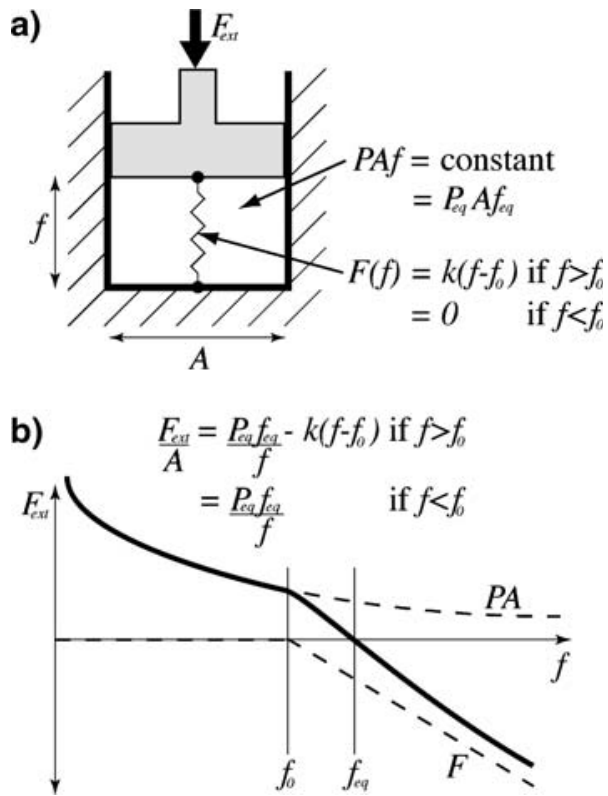

Fig. 6 Conceptual interpretation of high compressive moduli at low strains in cartilage-like materials. a Consider an isothermal ideal gas in a 1D piston chamber, wherein a piecewise linear spring of resting length $f_{0}$ resists upward movement of the massless piston (gray). At "free-swelling" equilibrium, where the external force $F_{\text {ext }}=0$, the spring extends to length $f_{\text {eq }}>f_{0}$. b Consideration of forces on the piston provides a "constitutive law" similar to present results. When the spring is stiffer than the gas, the modulus for small compressive strains where $f_{0}<f<f_{\text {eq }}$ may be greater than for larger strains where $f<f_{0}$

proposed interpretation of these results suggested that directions of increased tensile stiffness corresponding to preferentially oriented collagen fibrils (the split-line direction) were under increased pre-stress prior to compression (Mizrahi et al. 1986). The present model suggests the opposite: with isotropic PG gel swelling pressure less pre-stress is expected within individual fibers oriented along preferential directions. An alternate explanation for the observed behavior may simply be that more fibrils tend to be oriented along the split-line direction, making the collagen network stiffer along that direction. Further observations highlighted greater effects on tensile stiffness along the split-line direction for changes in ionic strength (PG gel swelling pressure) (Mizrahi et al. 1986). As illustrated in the present model, collagen network tensile deformations may occur due to fibril reorientation or fibril elongation, with greater stiffness associated with the latter case. It is therefore possible for directions of predominant fibril orientation to exhibit less stiffness than other directions, when fibril reorientation is the main consequence of matrix deformations in the predominant fibril direction while fibril elongation occurs in other directions.

The present study was restricted to equilibrium matrix mechanics and relatively simple physics of the PG gel in order to highlight novel features of the collagen network modeling approach. Future efforts could extend this work to address more general situations. Poroelastic mechanics during dynamic matrix deformations could be addressed similarly to

previous studies (Morel and Quinn 2004a) with the introduction of a matrix hydraulic permeability tensor given by $\gamma \kappa_{\mathrm{pg}}$, where $\kappa_{\text {pg }}$ represents the microstructure-dependent hydraulic permeability tensor of the PG gel (Quinn et al. 2001a). Contributions of the collagen network in other loading geometries could be investigated. For example, previous studies have investigated the contribution of changes in electrostatic energy density associated with glycosaminoglycan architecture to the cartilage shear modulus (Jin and Grodzinky 2001), but more careful attention to shear-induced changes in molecular architecture (Quinn 1996; Quinn et al. 2001a) indicates that the modeled effects were likely overestimated. The present model could be used to explore other contributions of the PG gel to cartilage shear mechanics, such as via induced pre-stresses in the collagen network. Mechanical interactions between cartilage zones and molecular-scale stress distributions resulting from inhomogeneous or discontinuous matrix structure might be explored in relation to full-thickness tissue function and interactions between cartilage-like tissues in tissue engineering or repair contexts. Mechanical failure of cartilage in compression likely results from matrix deformations which induce molecular-scale stresses exceeding some collagen fibril or network yield stress. Under high strain rate injurious compression, these phenomena are linked to fluid pressurization and can give rise to zone-specific mechanical damage (Morel and Quinn 2004b). The present modeling approach provides means to investigate loading of individual collagen fibrils for a range of matrix compositions and microstructures, which may help identify tissue loading environments which can induce collagen microdamage. More sophisticated fibril orientation distributions could also be investigated, in order to clarify the mechanical significance of specialized collagen architectures as in, for example, the split-line pattern of collagen orientation in the superficial zone.

\section{Appendix A: Symbols}

$\alpha$

A

$A_{\text {tot }}$ col

$\gamma \quad$ Volume fraction of PG gel within a matrix element

$d_{\text {tot }} \quad$ Length of imaginary control volume

$\varepsilon_{f} \quad$ Fibril tensile strain relative to zero-stress relaxed length

f Vector representing collagen fibril length and direction

i Unit vector

I Unit dyadic tensor

J Elastic stiffness tensor

K Hydraulic permeability tensor

n Unit normal vector to imaginary control surface

$N_{i} \quad$ Number of collagen fibrils with orientation labeled by $i$ 


\begin{tabular}{|c|c|}
\hline$N_{\text {tot }}$ & umber of collagen fibrils in a control volume \\
\hline pg & Referring to the proteoglycan gel \\
\hline$P$ & Orientation distribution of collagen fibrils \\
\hline $\mathbf{r}$ & Radial vector in spherical coordinates \\
\hline$R, \theta, \phi$ & Spherical coordinates $(0 \leq \theta \leq \pi ; 0 \leq \phi \leq 2 \pi)$ \\
\hline $\mathbf{R}$ & $\begin{array}{l}\text { Tensor describing rotation-induced changes in } \\
\text { (Eq. } 2 \text { and } 13 \text { ) }\end{array}$ \\
\hline$\sigma$ & Tensile stress in individual collagen fibrils \\
\hline SHD & Spherical harmonic decomposition \\
\hline $\mathbf{S}$ & $\begin{array}{l}\text { Equilibrium elastic stress tensor of a matrix ele- } \\
\text { ment }\end{array}$ \\
\hline $\mathbf{T}$ & $\begin{array}{l}\text { Tensor describing deformation-induced changes } \\
\mathbf{S} \text { (Eq. 13) }\end{array}$ \\
\hline & Tissue-scale vector displacement field \\
\hline
\end{tabular}

Acknowledgements This work was supported by a grant from the AO Research Foundation, Switzerland.

\section{References}

Alhadlaq HA, Xia Y (2004) The structural adaptations in compressed articular cartilage by microscopic MRI (microMRI) T(2) anisotropy. Osteoarthr Cartil 12(11):887-894

Armstrong CG, Lai WM, Mow VC (1984) An analysis of the unconfined compression of articular cartilage. J Biomech Eng 106:165-173

Barocas VH, Tranquillo RT (1997) An anisotropic biphasic theory of tissue-equivalent mechanics: the interplay among cell traction, fibrillar network deformation, fibril alignment, and cell contact guidance. J Biomech Eng 119(2):137-145

Bashir A, Gray ML, Hartke J, Burstein D (1999) Nondestructive imaging of human cartilage glycosaminoglycan concentration by MRI. Magn Reson Med 41(5):857-865

Benninghoff A (1925) Form und bau der gelenkknorpel in ihren beziehungen zur funktion II. Der aufbau des gelenkknorpels in seinen beziehungen zur funktion. Z Zellforsch Mikrosk Anat 2:783-862

Billiar KL, Sacks MS (2000) Biaxial mechanical properties of the native and glutaraldehyde-treated aortic valve cusp: Part II-A structural constitutive model. J Biomech Eng 122(4):327-335

Brenner H, Edwards DA (1993) Macrotransport Processes. Butterworth, Stoneham

Buschmann MD, Grodzinsky AJ (1995) A molecular model of proteoglycan-associated electrostatic forces in cartilage mechanics. J Biomech Eng 117:179-192

Camacho NP, West P, Torzilli PA, Mendelsohn R (2001) FTIR microscopic imaging of collagen and proteoglycan in bovine cartilage. Biopolymers $62(1): 1-8$

Chen AC, Bae WC, Schinagl RM, Sah RL (2001) Depth- and straindependent mechanical and electromechanical properties of fullthickness bovine articular cartilage in confined compression. J Biomech 34(1):1-12

Driessen NJ, Peters GW, Huyghe JM, Bouten CV, Baaijens FP (2003) Remodeling of continuously distributed collagen fibres in soft connective tissues. J Biomech 36(8):1151-1158

Eisenberg SR, Grodzinsky AJ (1988) Electrokinetic micromodel of extracellular matrix and other polyelectrolyte networks. Physicochemical Hydrodynamics 10:517-539

Farquhar T, Dawson PR, Torzilli PA (1990) A microstructural model for the anisotropic drained stiffness of articular cartilage. J Biomech Eng 112:414-425

Frank EH, Grodzinsky AJ (1987) Cartilage electromechanics I- electrokinetic transduction and the effects of electrolyte $\mathrm{pH}$ and ionic strength. J Biomech 20(6):615-627

Grodzinsky AJ (1983) Electromechanical and physicochemical properties of connective tissues. CRC Criti Rev Biomed Eng 9(2):133-199

Happel J (1959) Viscous flow relative to arrays of cylinders. AIChE Journal 5:174-177
Hunziker EB, Michel M, Studer D (1997) Ultrastructure of adult human articular cartilage matrix after cryotechnical processing. Microsc Res Tech 37(4):271-284

Jeffery AK, Blunn GW, Archer CW, Bentley G (1991) Threedimensional collagen architecture in bovine articular cartilage. J Bone Joint Surg Br 73(5):795-801

Jin M, Grodzinky AJ (2001) Effect of electrostatic interactions between glycosaminoglycans on the shear stiffness of cartilage: a molecular model and experiments. Macromolecules 34:8330-8339

Jurvelin JS, Buschmann MD, Hunziker EB (1997) Optical and mechanical determination of poisson's ratio of adult bovine humeral articular cartilage. J Biomech 30(3):235-241

Kääb MJ, Ito K, Clark JM, Notzli HP (1998) Deformation of articular cartilage collagen structure under static and cyclic loading. J Orthop Res 16(6):743-751

Khalsa P, Eisenberg SR (1997) Compressive behavior of articular cartilage is not completely explained by proteoglycan osmotic pressure. J Biomech 30(6):589-594

Kim YJ, Bonassar LJ, Grodzinsky AJ (1995) The role of cartilage streaming potential, fluid flow, and pressure in the stimulation of chondrocyte biosynthesis during dynamic compression. J Biomech 28(9):1055-1066

Kuwabara S (1959) The forces experienced by randomly distributed parallel circular cylinders or spheres in a viscous flow at small Reynolds numbers. J Physical Soc Japan 14:527-532

Li LP, Buschmann MD, Shirazi-Adl A (2000) A fibril reinforced nonhomogeneous poroelastic model for articular cartilage: inhomogeneous response in unconfined compression. J Biomech 33(12):1533-1541

Luo Z, Sun Y, Fujii T, An K (2004) Single molecule mechanical properties of type II collagen and hyaluronan measured by optical tweezers. Biorheology 41(3-4):247-254

Malvern L (1969) Introduction to the mechanics of a continuous medium. Prentice-Hall, Englewood Cliffs

Maroudas A (1976) Balance between swelling pressure and collagen tension in normal and degenerate cartilage. Nature 260:808-809

Maroudas A, Bannon C (1981) Measurement of swelling pressure in cartilage and comparison with the osmotic pressure of constituent proteoglycans. Biorheology 18(3-6):619-632

Maroudas A, Muir H, Wingham J (1969) The correlation of fixed negative charge with glycosaminoglycan content of human articular cartilage. Biochim Biophys Acta 177:492-500

Maroudas A, Wachtel E, Grushko G, Katz EP, Weinberg P (1991) The effect of osmotic and mechanical pressures on water partitioning in articular cartilage. Biochim Biophys Acta 1073:285-294

Mizrahi J, Maroudas A, Lanir Y, Ziv I, Webber TJ (1986) The instantaneous deformation of cartilage: effects of collagen fiber orientation and osmotic stress. Biorheology 23:311-330

Mollenhauer J, Aurich M, Muehleman C, Khelashvilli G, Irving TC (2003) X-ray diffraction of the molecular substructure of human articular cartilage. Connect Tissue Res 44(5):201-207

Morel V, Quinn TM (2004a) Short-term changes in cell and matrix damage following mechanical injury of articular cartilage explants and modeling of microphysical mediators. Biorheology 41(3-4):509-519

Morel V, Quinn TM (2004b) Cartilage injury by ramp compression near the gel diffusion rate. J Orthop Res 22(1):145-151

Mow VC, Holmes MH, Lai WM (1984) Fluid transport and mechanical properties of articular cartilage: a review. J Biomech 17(5):377-394

Park S, Krishnan R, Nicoll SB, Ateshian GA (2003) Cartilage interstitial fluid load support in unconfined compression. J Biomech 36(12):1785-1796

Pins G, Huang E, Christiansen D, Silver F (1997) Effects of static axial strain on the tensile properties and failure mechanisms of self-assembled collagen fibers. J Appl Polym Sci 63(11):1429-1440

Quinn TM, Dierickx P, Grodzinsky AJ (2001a) Glycosaminoglycan network geometry may contribute to anisotropic hydraulic permeability in cartilage under compression. J Biomech 34(11):1483-1490

Quinn TM, Morel V, Meister JJ (2001b) Static compression of articular cartilage can reduce solute diffusivity and partitioning: implications for the chondrocyte biological response. J Biomech 34(11):14631469 
Reynaud B, Quinn TM (2006) Anisotropic hydraulic permeability in compressed articular cartilage. J Biomechan 39(1):131-137

Seog J, Dean D, Plaas A, Wong-Palms S, Grodzinsky A, Ortiz C (2002) Direct measurement of glycosaminoglycan intermolecular interactions via high-resolution force spectroscopy. Macromolecules 35:5601-5615

Soulhat J, Buschmann MD, Shirazi-Adl A (1999) A fibril-networkreinforced biphasic model of cartilage in unconfined compression. J Biomech Eng 121(3):340-347

Torzilli PA, Arduino JM, Gregory JD, Bansal M (1997) Effect of proteoglycan removal on solute mobility in articular cartilage. J Biomechan 30(9):895-902

Williams JM, Uebelhart D, Thonar EJ, Kocsis K, Modis L (1996) Alteration and recovery of the spatial orientation of the collagen network of articular cartilage in adolescent rabbits following intra-articular chymopapain injection. Connect Tissue Res 34(2):105-117
Wilson W, van Donkelaar CC, van Rietbergen B, Ito K, Huiskes R (2004) Stresses in the local collagen network of articular cartilage: a poroviscoelastic fibril-reinforced finite element study. J Biomech 37(3):357-366

Wilson W, van Donkelaar CC, van Rietbergen B, Huiskes R (2005) A fibril-reinforced poroviscoelastic swelling model for articular cartilage. J Biomech 38(6):1195-1204

Xia Y, Elder K (2001) Quantification of the graphical details of collagen fibrils in transmission electron micrographs. J Microsc 204 (Pt 1):3-16

Xia Y, Moody JB, Burton-Wurster N, Lust G (2001) Quantitative in situ correlation between microscopic MRI and polarized light microscopy studies of articular cartilage. Osteoarthr Cartil 9(5):393-406

Zamparo O, Comper WD (1989) Hydraulic conductivity of chondroitin sulfate proteoglycan solutions. Arch Biochem Biophys 274(1): 259-269 